\title{
Truncation of the Earth Impulse Responses Relating Geoelectric Fields and Geomagnetic Field Variations
}

\author{
Risto J. Pirjola ${ }^{1,2}$ and David H. Boteler ${ }^{1}$ \\ ${ }^{1}$ Geomagnetic Laboratory, Earth Science Sector, Natural Resources Canada, 2617 Anderson Road, Ottawa, \\ Ontario, K1A 0E7, Canada \\ ${ }^{2}$ Finnish Meteorological Institute, P. O. Box 503, FI-00101 Helsinki, Finland \\ Email: risto.pirjola@fmi.fi
}

\begin{abstract}
To assess the geomagnetic hazard to power systems, it is necessary to model the Geomagnetically Induced Currents (GIC) produced during space weather storms. This requires knowledge of the geoelectric fields that drive GIC. In the time domain, the geoelectric fields can be calculated using a convolution integral including the geomagnetic field or its time derivative and an impulse response function for the Earth. In principle, the integral extends to infinity but for practical calculations the impulse responses must be truncated at a finite length. In this paper, we investigate the effects of the truncation on the calculation of the geoelectric fields. We consider how long the impulse responses need to be to obtain sufficiently accurate geoelectric field values. It is found that the high-pass impulse response used with geomagnetic data can be truncated very early, e.g. at $1 \mathrm{~h}$, while the low-pass impulse response used with geomagnetic time derivative data must be extended much longer, e.g. until $24 \mathrm{~h}$.
\end{abstract}

Keywords: Geoelectromagnetics, geoelectric field, Geomagnetically Induced Currents (GIC), space weather

\section{Introduction}

Geomagnetic disturbances can cause a variety of problems for power systems including damage to transformers $[1,2,3,4]$, misoperation of protective relays [5] and voltage stability problems [6]. Assessment of the geomagnetic hazard requires knowledge of the Geomagnetically Induced Currents (GIC) produced in a power network [7]. Modelling GIC involves two steps: i) use of geomagnetic field measurements with a model of the Earth's conductivity to calculate the geoelectric field at the Earth's surface, and ii) use of the geoelectric field values with a model of the power network to calculate the GIC [8].

Calculation of the geoelectric field can be performed either in the frequency domain or in the time domain. The frequency-domain method involves the Fourier transform of the geomagnetic field variations measured in the time domain to obtain the geomagnetic field spectrum, which is multiplied by the frequency-dependent transfer function of the Earth to get the geoelectric field spectrum. Finally, taking the inverse Fourier transform of the geoelectric spectrum gives the geoelectric field in the time domain. In the time-domain method, the geoelectric field is calculated from a convolution between the geomagnetic field variation and an impulse response function, which is related to the transfer function of the Earth. This paper is focussed on the time-domain calculation of the geoelectric field.

In the convolution used to calculate the geoelectric field, we can use either the geomagnetic field or its time derivative. In the frequency domain, the transfer function between the geoelectric and geomagnetic fields has the characteristics of a high-pass filter. Therefore, the impulse response included in the timedomain convolution with the geomagnetic field is called the "high-pass impulse response". On the other hand, the frequency-domain transfer function between the geoelectric field and the geomagnetic time derivative has the characteristics of a low-pass filter, and so the impulse response included in the timedomain convolution with the time derivative of the geomagnetic field is called the "low-pass impulse response". Based on the differential theorem of convolution, the high-pass impulse response is the time derivative of the low-pass impulse response. 
Theoretically the convolutions are integrals extending from minus infinity to plus infinity. However, the geoelectric field at a given time is only affected by geomagnetic variations before this time, which is equivalent to saying that the impulse responses are causal functions. This implies that we only integrate from zero to plus infinity in the convolutions, in which the variable of integration equals the time lag between the geoelectric and geomagnetic field. In practice, however, the integration is carried out only over a finite interval. In other words, the high-pass and low-pass impulse responses are truncated. In this paper, we investigate the effect of this truncation on the geoelectric field results.

In Section 2, we summarise the convolution integrals enabling the calculation of the geoelectric field from geomagnetic data. Section 3 is devoted to the mathematical theory associated with the truncation of the convolution integrals. The impulse responses for the special case of an Earth model with uniform conductivity are summarised in Section 4. For each frequency component of the geomagnetic field variation and a uniform-Earth model, the geoelectric field values obtained using truncated impulse responses have analytic expressions in terms of the Fresnel Integrals. This is investigated in Section 5. Calculations based on an artificial test geomagnetic variation waveform are considered in Section 6 . Conclusions are given in Section 7.

\section{Calculation of the Geoelectric Field}

Let us model the Earth as a half-space with a flat surface. We assume that the geoelectric and geomagnetic fields are spatially uniform in the horizontal direction ("plane wave assumption") and that they have a time $(t)$ dependence expressed by $e^{i \omega t}=e^{i 2 \pi f t}$ where $f$ is the frequency and $\omega=2 \pi f$ is the angular frequency. Following the choice recommended in [9] for geoscience applications, we mostly use the frequency, instead of the angular frequency, in this paper. Thus, regarding the quantities as functions of $f$, the final solutions are obtained by summing over all frequencies involved. At the Earth's surface, a horizontal geoelectric field component $E(f)$ can be expressed in terms of the perpendicular horizontal geomagnetic field component $B(f)$ as follows

$$
E(f)=K(f) B(f)
$$

where the transfer function $K(f)$ depends on frequency and the conductivity structure of the Earth. The geoelectric field $E(f)$ can also be written in the form $E(f)=Z(f) H(f)$ in terms of the surface impedance $Z(f)$ and the magnetic field strength $H(f)$. The relation between $B(f)$ and $H(f)$ is given by $B(f)=\mu_{0} H(f)$ where $\mu_{0}=4 \pi \cdot 10^{-7} \mathrm{H} / \mathrm{m}$ is the vacuum permeability. Thus, comparing with

equation (1) shows that $K(f)=\frac{Z(f)}{\mu_{0}}$. However, geomagnetic measurements produce values of $B($ not $H)$, so for practical calculations, we use formula (1). In equation (1), the directions of the $E(f)$ and $B(f)$ form a right-handed system together with the downward vertical direction. In geoelectromagnetics (as in this paper), the Earth's permeability is generally assumed to equal $\mu_{0}$, and due to the low frequencies involved, the displacement currents can be neglected, and so the permittivity of the Earth does not play any role.

Equation (1) directly suggests how the geoelectric field $E(f)$ in the time domain can be calculated from time-domain geomagnetic variations $B(f)$, i.e.

1) Fourier transform $B(t)$ to obtain $B(f)$

2) Multiply $B(f)$ by $K(f)$ to obtain $E(f)$

3) Inverse Fourier transform $E(f)$ to obtain $E(t)$

This is the so-called "frequency-domain method" [10, 11].

Utilising the convolution theorem, which states that multiplication in the frequency domain corresponds to convolution in the time domain, equation (1) leads to the time-domain relation between $E(t)$ and $B(t)$ as follows

$$
E(t)=\int_{-\infty}^{\infty} R(\tau) B(t-\tau) d \tau
$$

where the impulse response $R(\tau)$ is the inverse Fourier transform of the transfer function $K(f)$, i.e. 


$$
R(\tau)=\int_{-\infty}^{\infty} K(f) e^{i 2 \pi f \tau} d f
$$

The absolute value of the transfer function $|K(f)|$ increases with increasing $f$, which means that $K(f)$ has the characteristics of a high-pass filter. Therefore $R(\tau)$ is called the "high-pass impulse response".

Denoting the time derivative of $B(t)$ by $g(t)$, the Fourier transform $g(f)$ is simply obtained by multiplying $B(f)$ by $i 2 p f$. Consequently, equation (1) can be written as

$$
E(f)=C(f) g(f)
$$

where

$$
C(f)=\frac{K(f)}{i 2 \pi f}
$$

In magnetotellurics, $C(f)$ defined as $\frac{E(f)}{g(f)}$ is called the "magnetotelluric relation". It should be noted that $C(f)$ is equal to the "complex skin depth" denoted by $p(f)$ and used for other purposes (see e.g. [12]).

Similarly to formula (2), equation (4) leads to a time-domain convolution relation between $E(t)$ and $g(t)$ as follows

$$
E(t)=\int_{-\infty}^{\infty} S(\tau) g(t-\tau) d \tau
$$

where the impulse response $S(t)$ is the inverse Fourier transform of the magnetotelluric relation $C(f)$, i.e.

$$
S(\tau)=\int_{-\infty}^{\infty} C(f) e^{i 2 \pi f \tau} d f
$$

The absolute value of the magnetotelluric relation $|C(f)|$ decreases with increasing $f$, which means that $C(f)$ has the characteristics of a low-pass filter. Therefore $S(\tau)$ is called the "low-pass impulse response".

Substituting $g(t-\tau)=\frac{d B(t-\tau)}{d(t-\tau)}=-\frac{d B(t-\tau)}{d \tau}$ into equation (6), performing a partial integration and comparing the result with equation (2), we see that

$$
R(\tau)=\frac{d S(\tau)}{d \tau}
$$

where we also make the natural assumption that $S(\tau) B(t-\tau)=0$ when $\tau=-\infty$ and $\tau=+\infty$, i.e. either $S(\tau)$ or $B(t-\tau)$ (or both) vanishes for $\tau=-\infty$ and for $\tau=+\infty$. Equation (8) is actually a result from the differential theorem of convolution.

For physical reasons, it is clear that the geoelectric field at a given time $t$ can only be affected by the geomagnetic field values earlier than $t$. This requirement is satisfied since the impulse responses $R(\tau)$ and $S(\tau)$ included in formulas (2) and (6) are causal functions, i.e. zero for negative values of the argument $\tau$, which is the time lag between geoelectric and geomagnetic variation data. Consequently, the integration limits can be set from 0 to $+\infty$ in equations (2) and (6), instead of integrating from $-\infty$ to $+\infty$.

\section{Mathematical Formulation of the Truncation of the Impulse Responses}

In practical calculations of the geoelectric field $E(t)$ from geomagnetic variations $B(t)$, the integrals appearing in equations (2) and (6) can only be taken over a finite interval. In other words, the impulse response functions are truncated, instead of letting them be non-zero until infinity. In this paper, we investigate the effect of the truncation on the calculated geoelectric field values. (Another practical thing 
in numerical calculations is that equations (2) and (6) must be approximated by sums but investigating this does not belong to the scope of this paper.)

Equations (2) and (6) give the exact geoelectric field denoted by $E_{\text {exact }}(t)$. As mentioned in Section 2, due to causality, the integrals need not be extended from $-\infty$ to $+\infty$, but the integration limits can be set from 0 to $+\infty$. This is, however, an irrelevant matter in the investigation of the effect of the truncation. Thus, in this section, we keep the integrals from $-\infty$ to $+\infty$, which also makes the study more general and applicable to any functions, not only to those associated with geoelectric and geomagnetic fields.

Let us consider the geoelectric fields obtained from formulas (2) and (6) when $R(\tau)$ and $S(\tau)$ are replaced with the impulse responses $R_{\text {trunc }}(\tau)$ and $S_{\text {trunc }}(\tau)$ truncated at $\tau=t_{0}$, i.e. $R_{\text {trunc }}(\tau)$ and $S_{\text {trunc }}(\tau)$ are zero when $\tau>t_{0}$ and equal to $R(\tau)$ and $S(\tau)$, respectively, when $\tau<t_{0}$. Expressing this mathematically gives

$$
\begin{aligned}
& R_{\text {trunc }}(\tau)=R(\tau)\left(1-\Theta\left(\tau-t_{0}\right)\right) \\
& S_{\text {trunc }}(\tau)=S(\tau)\left(1-\Theta\left(\tau-t_{0}\right)\right)
\end{aligned}
$$

Where $\Theta\left(\tau-t_{0}\right)$ is the Heaviside step function being zero when $\tau<t_{0}$ and +1 when $\tau>t_{0}$. (Note that alternatively the function $1-\Theta\left(\tau-t_{0}\right)$ could also be expressed as $\Theta\left(t_{0}-\tau\right)$.) Substituting $R_{\text {trunc }}(\tau)$ and $S_{\text {trunc }}(\tau)$ from formulas $(9)$ and (10) for $R(\tau)$ and $S(\tau)$ in equations (2) and (6) results in $E_{\text {trunc_B }}(t)$ and $E_{\text {trunc_g }}(t)$ given by

$$
\begin{aligned}
& E_{\text {trunc_B }}(t)=\int_{-\infty}^{\infty} R_{\text {trunc }}(\tau) B(t-\tau) d \tau \\
& =\int_{-\infty}^{t_{0}} R(\tau) B(t-\tau) d \tau \\
& =E_{\text {exact }}(t)-\int_{t_{0}}^{\infty} R(\tau) B(t-\tau) d \tau \\
& E_{\text {trunc_g }}(t)=\int_{-\infty}^{\infty} S_{\text {trunc }}(\tau) g(t-\tau) d \tau \\
& =\int_{-\infty}^{t_{0}} S(\tau) g(t-\tau) d \tau \\
& =E_{\text {exact }}(t)-\int_{t_{0}}^{\infty} S(\tau) g(t-\tau) d \tau
\end{aligned}
$$

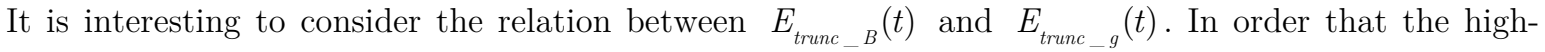
pass (equation (2)) and low-pass (equation (6)) calculations lead to the same geoelectric field values, the impulse responses must satisfy equation (8). This suggests that we should look at the derivative of $S_{\text {trunc }}(\tau)$. Using the derivative rule of a product, the fact that the derivative of the Heaviside step function $\Theta\left(\tau-t_{0}\right)$ equals the Dirac delta function $\delta\left(\tau-t_{0}\right)$ and equations (8), (9) and (10), we obtain

$$
\begin{aligned}
& \frac{d S_{\text {trunc }}(\tau)}{d \tau}=\frac{d S(\tau)}{d \tau}\left(1-\Theta\left(\tau-t_{0}\right)\right)-S(\tau) \delta\left(\tau-t_{0}\right) \\
& =R(\tau)\left(1-\Theta\left(\tau-t_{0}\right)\right)-S(\tau) \delta\left(\tau-t_{0}\right) \\
& =R_{\text {trunc }}(\tau)-S(\tau) \delta\left(\tau-t_{0}\right)
\end{aligned}
$$

Formula (13) shows that $R_{\text {trunc }}(\tau) \neq \frac{d S_{\text {trunc }}(\tau)}{d \tau}$ because $S\left(t_{0}\right)$ not zero. Thus $E_{\text {trunc }}(t)$ and $E_{\text {trunc_g }}(t)$ are not equal. 
Let us define another truncated high-pass impulse response $R_{\text {tr* }}(\tau)=\frac{d S_{\text {trunc }}(\tau)}{d \tau}=R_{\text {trunc }}(\tau)-S(\tau) \delta\left(\tau-t_{0}\right)$. Substituting $R_{t r^{*}}(\tau)$ for $R(\tau)$ in equation (2) and using formula (11) result in $E_{t r^{*}{ }_{B}}(t)$ given by

$$
\begin{aligned}
& E_{{\text {tr }{ }_{-} B}}(t)=E_{\text {trunc_B }}(t)-\int_{-\infty}^{\infty} S(\tau) \delta\left(\tau-t_{0}\right) B(t-\tau) d \tau \\
& =E_{\text {trunc }_{-} B}(t)-S\left(t_{0}\right) B\left(t-t_{0}\right) \\
& =E_{\text {exact }}(t)-\int_{t_{0}}^{\infty} R(\tau) B(t-\tau) d \tau-S\left(t_{0}\right) B\left(t-t_{0}\right)
\end{aligned}
$$

Considering the term $-S\left(t_{0}\right) B\left(t-t_{0}\right)$ in greater detail, equation (14) shows that it results from the delta function term included in the definition of $R_{t r^{*}}(t)$, i.e. from the difference between $R_{t r^{*}}(t)$ and $R_{\text {trunc }}(t)$, and gives the difference between $E_{t r^{*} B}(t)$ and $E_{\text {trunc_B }}(t)$. Assuming that $S(\tau) B(t-\tau)=0$ when $\tau=+\infty$, i.e. either $S(\tau)$ or $B(t-\tau)$ (or both) vanishes for $\tau=+\infty$, which is a very natural assumption (see the remarks after equation (8)), we can express the term $-S\left(t_{0}\right) B\left(t-t_{0}\right.$ ) as follows

$$
\begin{aligned}
& -S\left(t_{0}\right) B\left(t-t_{0}\right)=\int_{t_{0}}^{\infty} \frac{d}{d \tau}(S(\tau) B(t-\tau)) d \tau \\
& =\int_{t_{0}}^{\infty} \frac{d S(\tau)}{d \tau} B(t-\tau) d \tau+\int_{t_{0}}^{\infty} S(\tau) \frac{d B(t-\tau)}{d \tau} d \tau \\
& =\int_{t_{0}}^{\infty} R(\tau) B(t-\tau) d \tau-\int_{t_{0}}^{\infty} S(\tau) g(t-\tau) d \tau
\end{aligned}
$$

where the derivative rule of a product, equation (8) and the fact that $g(t)$ is the time derivative of $B(t)$ are used. Substituting formula (15) into equation (14) yields

$$
E_{\text {tr*_B }}(t)=E_{\text {exact }}(t)-\int_{t_{0}}^{\infty} S(\tau) g(t-\tau) d \tau
$$

Comparing equations (12) and (16) shows that $E_{\text {trunc }} g(t)$ and $E_{t r^{*}{ }_{B}}(t)$ are equal. This is expected due to the differential theorem of convolution and the definition of $R_{t r^{*}}(\tau)$. Formulas $(14)$ and (15) show that the delta function term included in $R_{t r *}(\tau)$ is due to the neglected integrals from $t_{0}$ to infinity when the truncated impulse responses are used in equations (11) and (12).

\section{Impulse Responses for a Uniform Earth}

If the Earth is modelled as a half-space with uniform conductivity $\sigma$ the transfer function $K(f)$ is

$$
K(f)=\sqrt{\frac{i 2 \pi f}{\mu_{0} \sigma}}
$$

(see e.g. [13], p. 21). Thus, based on equation (5), the magnetotelluric relation $C(f)$ is

$$
C(f)=\frac{1}{\sqrt{i 2 \pi f \mu_{0} \sigma}}=\frac{e^{-i \frac{\pi}{4}}}{\sqrt{2 \pi \mu_{0} \sigma}} \cdot \frac{1}{\sqrt{f}}
$$

Substitution of formula (18) into equation (7) gives the low-pass impulse response $S(t)$ for a uniform Earth. However, it should be noted that the integral in equation (7) contains both positive and negative values of the frequency $f$. Therefore, we have to carefully define how the square root of $f$ appearing in formula (18) is taken when $f<0$. This definition is dictated by the requirement that $S(t)$ must be a real function, which implies that, for all values of $f$, 


$$
C(f)=C^{*}(-f)
$$

where the asterisk $\left(^{*}\right)$ denotes the complex conjugate. Utilising equation (19), it follows from formula (18) that, for negative values of $f$, the square root of $f$, must lie on the negative imaginary axis, i.e. $\sqrt{f}=-i \sqrt{-f}$ when $f<0$ (cf. [13], p. 22). Consequently, equations (7) and (18) yield

$$
\begin{aligned}
& S(\tau)=\frac{e^{-i \frac{\pi}{4}}}{\sqrt{2 \pi \mu_{0} \sigma}}\left[\int_{0}^{\infty} \frac{e^{i 2 \pi f \tau}}{\sqrt{f}} d f+\int_{-\infty}^{0} \frac{e^{i 2 \pi f \tau}}{-i \sqrt{-f}} d f\right] \\
& =\frac{e^{-i \frac{\pi}{4}}}{\sqrt{2 \pi \mu_{0} \sigma}} \int_{0}^{\infty}\left(\frac{e^{i 2 \pi f \tau}}{\sqrt{f}}+i \frac{e^{-i 2 \pi f \tau}}{\sqrt{f}}\right) d f \\
& =\frac{e^{-i \frac{\pi}{4}}}{\sqrt{2 \pi \mu_{0} \sigma}}(1+i)\left[\int_{0}^{\infty} \frac{\cos (2 \pi f \tau)}{\sqrt{f}} d f+\int_{0}^{\infty} \frac{\sin (2 \pi f \tau)}{\sqrt{f}} d f\right]
\end{aligned}
$$

Using the formulas

$$
\begin{gathered}
\int_{0}^{\infty} \frac{\cos (\alpha u)}{\sqrt{u}} d u=\sqrt{\frac{\pi}{2|\alpha|}} \\
\int_{0}^{\infty} \frac{\sin (\alpha u)}{\sqrt{u}} d u=\operatorname{sign}(\alpha) \sqrt{\frac{\pi}{2|\alpha|}}
\end{gathered}
$$

where $\operatorname{sign}(a)$ equals +1 and -1 for positive and negative values of $\alpha$, respectively, we can immediately see that, for $\tau<0$, the $\cos$ and $\sin$ integrals cancel each other in equation (20) making $S(\tau)$ zero when $\tau<0$, which is in accordance with the causality of the impulse response. For $\tau>0$, the $\cos$ and $\sin$ integrals give the same contribution equal to $\frac{1}{2} \sqrt{\frac{1}{\tau}}$. Substituting this into equation (20), we can write $S(\tau)$ for all values of $\tau$ as

$$
S(\tau)=\frac{1}{\sqrt{\pi \mu_{0} \sigma \tau}} \Theta(\tau)
$$

where $\Theta(\tau)$ is the Heaviside step function being zero when $\tau<0$ and +1 when $\tau>0$.

The high-pass impulse response $R(\tau)$ for a uniform Earth can be found, following equation (8), by differentiating the low-pass impulse response $S(\tau)$ given by equation (23). Thus, using the derivative rule of a product and the fact that the derivative of the Heaviside step function $\Theta(\tau)$ is the Dirac delta function $\delta(\tau)$, we obtain

$$
\begin{aligned}
& R(\tau)=\frac{d S(\tau)}{d \tau}=\frac{d}{d \tau}\left(\frac{1}{\sqrt{\pi \mu_{0} \sigma \tau}} \Theta(\tau)\right)=\frac{1}{\sqrt{\pi \mu_{0} \sigma}}\left(\frac{1}{\sqrt{\tau}} \frac{d \Theta(\tau)}{d \tau}+\frac{d \tau^{-1 / 2}}{d \tau} \Theta(\tau)\right) \\
& =\frac{1}{\sqrt{\pi \mu_{0} \sigma \tau}}\left(\delta(\tau)-\frac{\Theta(\tau)}{2 \tau}\right)=\frac{\delta(\tau)}{\sqrt{\pi \mu_{0} \sigma \tau}}-\frac{S(\tau)}{2 \tau}
\end{aligned}
$$

Using a value of $0.001 \mathrm{~S} / \mathrm{m}$ for the Earth's conductivity, the high-pass and low-pass impulse responses $R(\tau)$ and $S(\tau)$ given by equations (24) and (23), respectively, are shown by the red lines in Figures 1a and $1 \mathrm{~b}$. We see that $R(\tau)$ goes to zero much faster than $S(\tau)$ with increasing $\tau$, which represents the time lag between geoelectric and geomagnetic data. Mathematically this is clear because $R(\tau)$ has a $\tau^{-3 / 2}$ dependence on $\tau$ whereas $S(\tau)$ follows $\tau^{-1 / 2}$. The faster decrease of $R(\tau)$ indicates that, in the convolution calculations, $R(\tau)$ can be truncated much earlier than $S(\tau)$. This will be investigated more in the next sections of this paper. The black lines in Figures 1a and 1b represent impulse responses truncated at $t_{0}=18 \mathrm{~min}$ and $t_{0}=2 \mathrm{~h} 24 \mathrm{~min}$, respectively. These values of $t_{0}$ are chosen arbitrarily just for the plots in question. 
High-Pass Impulse Response; Uniform Earth $1000 \mathrm{ohm} \mathrm{m;} \mathrm{Red=ImpResp,} \mathrm{Black=TruncatedImpResp}$

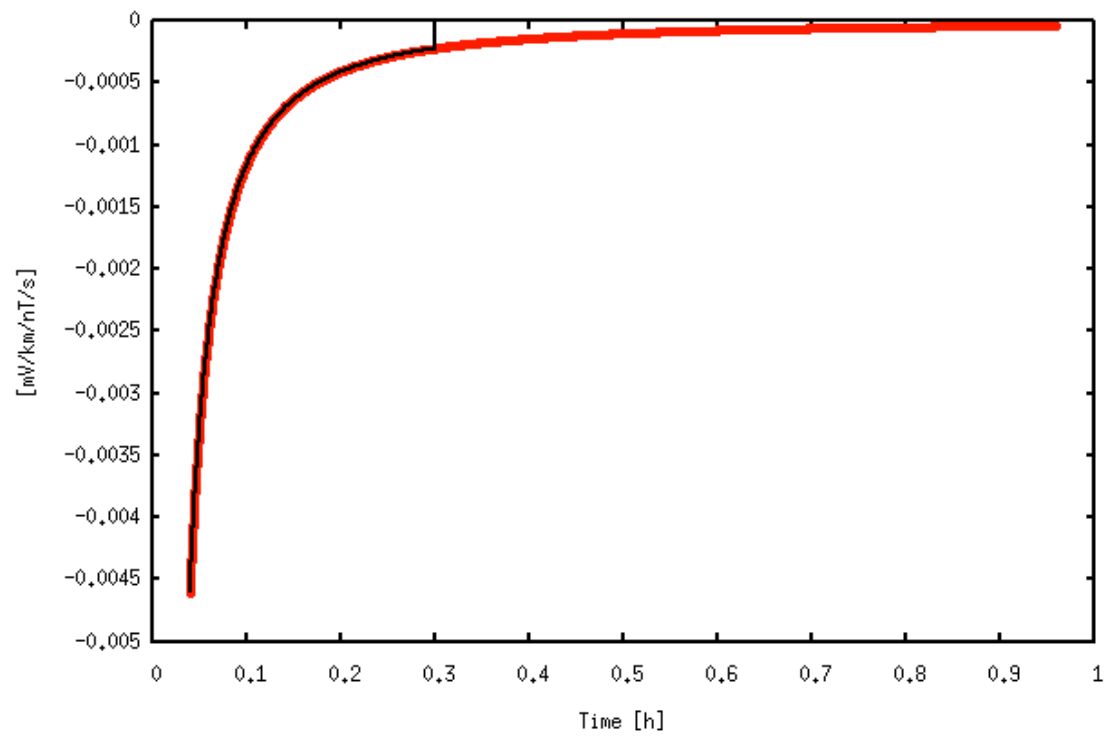

(a)

Low-Pass Impulse Response; Uniform Earth 1000 ohm m; Red=ImpResp, Black=TruncatedImpResp

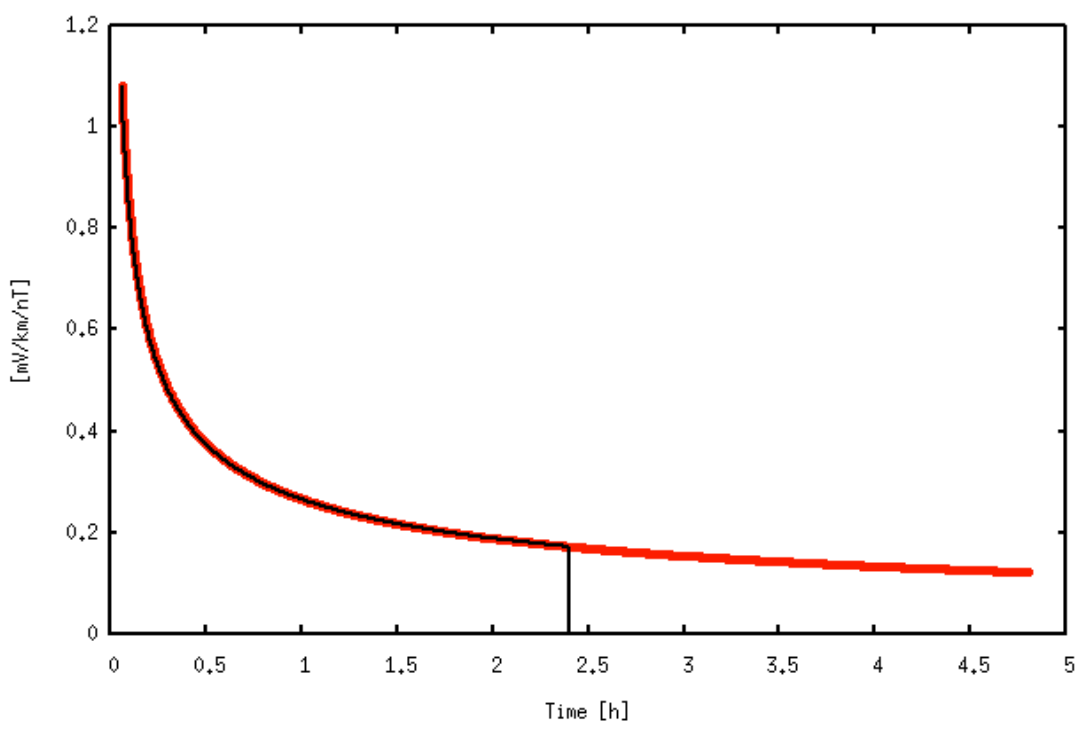

(b)

Figure 1. (a) High-pass impulse response for a uniform Earth given by equation (24) (red curve). The black curve shows the high-pass impulse response truncated at $t_{0}=18 \mathrm{~min}$. (b) Low-pass impulse response for a uniform Earth given by equation (23) (red curve). The black curve shows the low-pass impulse response truncated at $t_{0}=2 \mathrm{~h} 24$ min. In both plots, the Earth's conductivity is $\sigma=0.001 \mathrm{~S} / \mathrm{m}$.

\section{Calculation of the Geoelectric Field Due to a Sinusoidal Geomagnetic}

\section{Variation}

For a sinusoidal geomagnetic field variation $B(t)$ of the form

$$
B(t)=B_{0} \sin (\omega t+\phi)
$$

where $B_{0}, \omega$ and $\phi$ are the amplitude, the angular frequency and the phase angle, the time derivative $g(t)$ is 


$$
g(t)=\frac{d B(t)}{d t}=\omega B_{0} \cos (\omega t+\phi)
$$

In this section, we calculate the geoelectric field by convolving $B(t)$ or $g(t)$ with the appropriate impulse response. For an Earth modelled with a uniform conductivity $\sigma$, the high-pass and low-pass impulse responses are given by equations (24) and (23), respectively. These can then be substituted into equations (9) and (10) that give the truncated impulse responses, and the geoelectric field $E_{\text {trunc } \_B}(t)$ or $E_{\text {trunc_g }}(t)$ is given by equation (11) or (12) as follows

$$
\begin{gathered}
E_{\text {trunc_B}}(t)=\frac{B_{0}}{\sqrt{\pi \mu_{0} \sigma}}\left[\int_{-\infty}^{t_{0}} \frac{\delta(\tau) \sin (\omega(t-\tau)+\phi)}{\sqrt{\tau}} d \tau\right. \\
\left.-\frac{1}{2} \int_{0}^{t_{0}} \frac{\sin (\omega(t-\tau)+\phi)}{\tau \sqrt{\tau}} d \tau\right] \\
E_{\text {trunc_g }}(t)=\frac{\omega B_{0}}{\sqrt{\pi \mu_{0} \sigma}} \int_{0}^{t_{0}} \frac{\cos (\omega(t-\tau)+\phi)}{\sqrt{\tau}} d \tau
\end{gathered}
$$

We first consider equation (28), which is simpler. Applying the trigonometric formula $\cos (\kappa-\beta)=\cos (\kappa) \cos (\beta)+\sin (\kappa) \sin (\beta)$, equation (28) can be written as

$$
E_{\text {trunc }_{-} g}(t)=\frac{\omega B_{0}}{\sqrt{\pi \mu_{0} \sigma}}\left[\cos (\omega t+\phi) \int_{0}^{t_{0}} \frac{\cos (\omega \tau)}{\sqrt{\tau}} d \tau+\sin (\omega t+\phi) \int_{0}^{t_{0}} \frac{\sin (\omega \tau)}{\sqrt{\tau}} d \tau\right]
$$

For $t_{0}=\infty$, equation (29), with the application of formulas (21) and (22), the trigonometric formula $\sin (\kappa+\beta)=\sin (\kappa) \cos (\beta)+\cos (\kappa) \sin (\beta)$ and the fact that $\sin \left(\frac{\pi}{4}\right)=\cos \left(\frac{\pi}{4}\right)=\frac{1}{\sqrt{2}}$, results in the following exact expressions of the geoelectric field

$$
E_{\text {exact }}(t)=B_{0} \sqrt{\frac{\omega}{2 \mu_{0} \sigma}}[\cos (\omega t+\phi)+\sin (\omega t+\phi)]=B_{0} \sqrt{\frac{\omega}{\mu_{0} \sigma}} \sin \left(\omega t+\phi+\frac{\pi}{4}\right)
$$

This equation demonstrates the well-known 45-degree phase shift between the geoelectric and geomagnetic fields for a uniform Earth. If the phase angle of the geomagnetic variation $\phi$ is set to zero in equation (25), formula (30) gives the same result as presented in [14] (where a minus sign is included due to a difference in the coordinates).

To calculate the geoelectric field using an impulse response that is truncated, i.e. $t_{0}<\infty$, let us change the variable of integration from $\tau$ to $s$ in the two integrals in formula (29) by defining $\tau=\frac{\pi s^{2}}{2 \omega}$. Then the integrals appearing in equation (29) are

$$
\begin{aligned}
& \int_{0}^{t_{0}} \frac{\cos (\omega \tau)}{\sqrt{\tau}} d \tau=\sqrt{\frac{2 \pi}{\omega}} \int_{0}^{\sqrt{\frac{2 t_{0}}{\pi}}} \cos \left(\frac{\pi s^{2}}{2}\right) d s \\
& \int_{0}^{t_{0}} \frac{\sin (\omega \tau)}{\sqrt{\tau}} d \tau=\sqrt{\frac{2 \pi}{\omega}} \int_{0}^{\sqrt{\frac{2 \omega t_{0}}{\pi}}} \sin \left(\frac{\pi s^{2}}{2}\right) d s
\end{aligned}
$$

The integrals on the right-hand sides of equations (31) and (32) have the form of the Fresnel Cosine and Sine Integrals defined as

$$
\begin{aligned}
& F_{C}(\xi)=\int_{0}^{\xi} \cos \left(\frac{\pi s^{2}}{2}\right) d s \\
& F_{S}(\xi)=\int_{0}^{\xi} \sin \left(\frac{\pi s^{2}}{2}\right) d s
\end{aligned}
$$

where $\xi$ is any real or complex number. Denoting $\sqrt{\frac{2 \omega t_{0}}{\pi}}$ by $\eta$, equation (29) can thus be written as 


$$
E_{\text {trunc_g }}(t)=B_{0} \sqrt{\frac{2 \omega}{\mu_{0} \sigma}}\left[F_{C}(\eta) \cos (\omega t+\phi)+F_{S}(\eta) \sin (\omega t+\phi)\right]
$$

Describing the sinusoidal geomagnetic variation given by equation (25) in terms of the frequency $f=\frac{\omega}{2 \pi}$ and noting that the period $T$ equals the inverse of $f$, the variable $\eta$ can be expressed as $\eta=2 \sqrt{f t_{0}}=2 \sqrt{\frac{t_{0}}{T}}$, and $E_{\text {trunc } \_g}(t)$ gets the form

$$
E_{\text {trunc_g }}(t)=2 B_{0} \sqrt{\frac{\pi}{T \mu_{0} \sigma}}\left[F_{C}(\eta) \cos \left(\frac{2 \pi t}{T}+\phi\right)+F_{S}(\eta) \sin \left(\frac{2 \pi t}{T}+\phi\right)\right]
$$

If the argument $\xi$ in the definition of the Fresnel Integrals (equations (33) and (34)) has the value $+\infty$, both integrals equal $+\frac{1}{2}$. Thus, noting that $\eta=\infty$ when $t_{0}=\infty$ and substituting $+\frac{1}{2}$ for $F_{C}(\eta)$ and $F_{S}(\eta)$ in equation $(36)$, we see that, for $t_{0}=\infty, E_{\text {trunc } \_g}(t)$ reduces to $E_{\text {exact }}(t)$ given by equation $(30)$, as expected.

Next we consider equation (27), which gives $E_{\text {trunc } B}(t)$. Due to the delta function, the first integral is easy to calculate, even though it results in an infinite value. A partial integration can be applied to the second integral in equation (27). Consequently

$$
E_{\text {trunc_B }}(t)=\frac{B_{0}}{\sqrt{\pi \mu_{0} \sigma}}\left[\frac{\sin (\omega t+\phi)}{\sqrt{0}}+/ \frac{\sin (\omega(t-\tau)+\phi)}{\sqrt{\tau}}+\omega \int_{0}^{t_{0}} \frac{\cos (\omega(t-\tau)+\phi)}{\sqrt{\tau}} d \tau\right]
$$

As pointed out, the first term on the right-hand side of equation (37) is infinite. However, the lower limit in the second substitution term gives the same infinity but with an opposite sign, so these infinities cancel each other. Thus

$$
E_{\text {trunc_B }}(t)=\frac{B_{0}}{\sqrt{\pi \mu_{0} \sigma}} \frac{\sin \left(\omega\left(t-t_{0}\right)+\phi\right)}{\sqrt{t_{0}}}+\frac{\omega B_{0}}{\sqrt{\pi \mu_{0} \sigma}} \int_{0}^{t_{0}} \frac{\cos (\omega(t-\tau)+\phi)}{\sqrt{\tau}} d \tau
$$

The second term on the right-hand side of formula $(38)$ equals $E_{\text {trunc_g }}(t)$ (see equation $(28)$ ). Using the trigonometric formula $\sin (\kappa-\beta)=\sin (\kappa) \cos (\beta)-\cos (\kappa) \sin (\beta)$, equation (38) then yields

$$
E_{\text {trunc_B }}(t)=\frac{B_{0}}{\sqrt{\pi \mu_{0} \sigma}}\left[\frac{\cos \left(\omega t_{0}\right)}{\sqrt{t_{0}}} \sin (\omega t+\phi)-\frac{\sin \left(\omega t_{0}\right)}{\sqrt{t_{0}}} \cos (\omega t+\phi)\right]+E_{\text {trunc_g }_{-}}(t)
$$

We again use the frequency $f$ and the period $T$ to describe the sinusoidal geomagnetic variation, and define the variable $\eta=2 \sqrt{f t_{0}}=2 \sqrt{\frac{t_{0}}{T}}$ as above. Thus, utilising equation (36), equation (39) gives

$$
E_{\text {trunc_B }}(t)=2 B_{0} \sqrt{\frac{\pi}{T \mu_{0} \sigma}}\left[G_{C} \cos \left(\frac{2 \pi t}{T}+\phi\right)+G_{S} \sin \left(\frac{2 \pi t}{T}+\phi\right)\right]
$$

where the coefficients $G_{C}$ and $G_{S}$ are defined as

$$
\begin{aligned}
& G_{C}=G_{C}(\eta)=F_{C}(\eta)-\frac{1}{\pi \eta} \sin \left(\frac{\pi \eta^{2}}{2}\right) \\
& G_{S}=G_{S}(\eta)=F_{S}(\eta)+\frac{1}{\pi \eta} \cos \left(\frac{\pi \eta^{2}}{2}\right)
\end{aligned}
$$

For $t_{0}=\infty, \eta=\infty$, which makes the second terms on the right-hand side of formulas (41) and (42) vanish. Thus, since the Fresnel Integrals equal $+\frac{1}{2}$ for an infinite argument, $G_{C}=G_{S}=+\frac{1}{2}$ when $t_{0}=\infty$. Consequently, for $t_{0}=\infty$, the high-pass and low-pass calculations lead to the same geoelectric field values, which also equal $E_{\text {exact }}(t)$ given by equation (30), as expected. Comparing equation (40) with equation (36) and looking at formulas (41) and (42), we see that the second terms in the definition of $G_{C}$ 
and $G_{S}$ represent a kind of "conversion terms" if geoelectric field values obtained by convolving $g(t)=\frac{d B(t)}{d t}$ and the truncated low-pass impulse response are converted to geoelectric field values obtained by convolving $B(t)$ and the truncated high-pass impulse response.

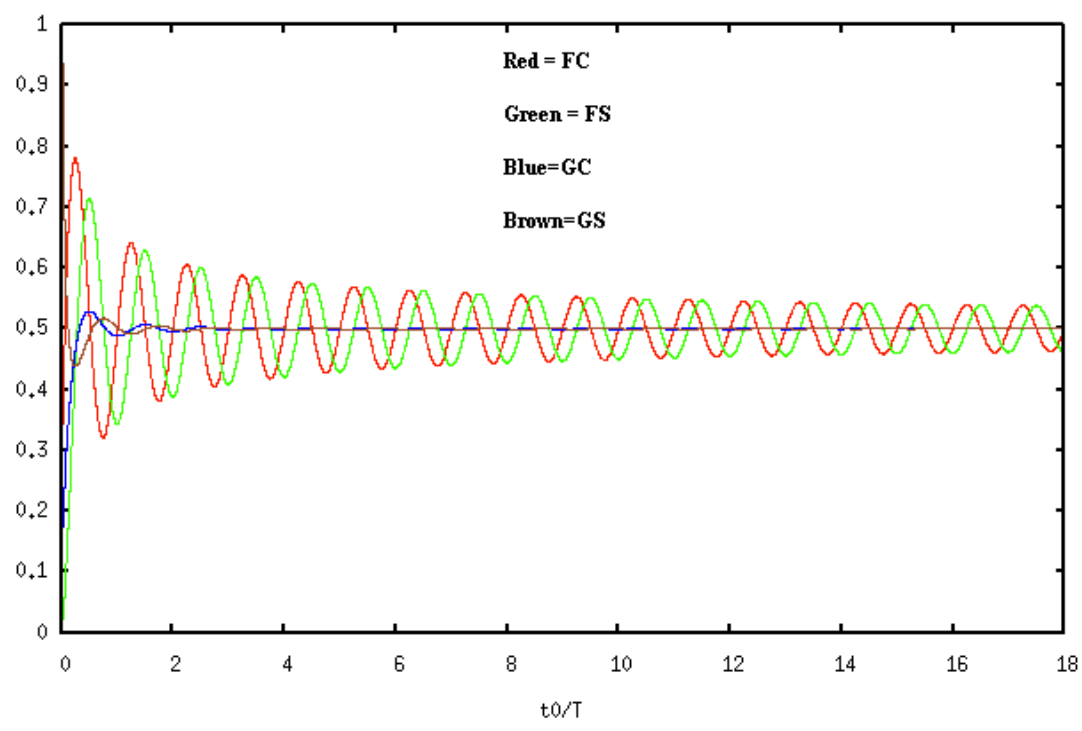

(a)

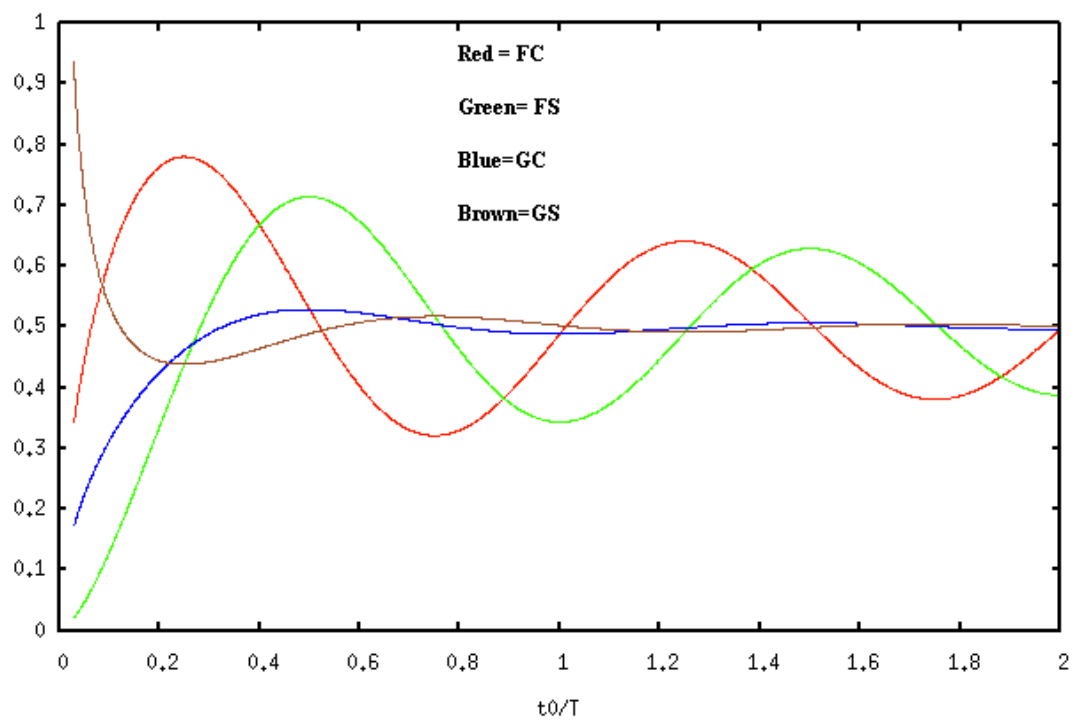

Figure 2. Fresnel Integrals $F_{C}$ (red) and $F_{S}$ (green) defined by equations (33) and (34) with $\xi=\eta=2 \sqrt{\frac{t_{0}}{T}} \square$ and included in equation (36) and coefficients $G_{C}$ (blue) and $G_{S}$ (brown) defined by equations (41) and (42) with $\eta=2 \sqrt{\frac{t_{0}}{T}}$ and included in equation (40) as functions of $\frac{t_{0}}{T}$ where $t_{0}$ is the impulse response length and $T$ is the period of the assumed sinusoidal variation of the geomagnetic field. Figure (b) is a zoomed-in view of the curves in Figure (a) at values of $\frac{t_{0}}{T}$ less than about 2 . 
Formula (36) shows that the closer $F_{C}$ and $F_{S}$ (having the implicit argument $\eta=2 \sqrt{\frac{t_{0}}{T}}$ ) are to the value $\frac{1}{2}$ the more accurate are the geoelectric field values obtained by using $g(t)=\frac{d B(t)}{d t}$ and the truncated low-pass impulse response. Similarly, formula (40) shows that the closer $G_{C}$ and $G_{S}$ are to the value $\frac{1}{2}$ the more accurate are the geoelectric field values obtained by using $B(t)$ and the truncated high-pass impulse response. Fig. 2 presents the coefficients $F_{C}$ (red), $F_{S}$ (green), $G_{C}$ (blue) and $G_{S}$ (brown) as functions of $\frac{t_{0}}{T}$. Fig. $2 \mathrm{~b}$ is a zoomed-in view of the curves shown in Fig. 2a at small values of $\frac{t_{0}}{T}$. We can see that all the quantities investigated approach $\frac{1}{2}$ when $\frac{t_{0}}{T}$ increases. This is an expected result since longer impulse responses should lead to more accurate geoelectric field values and, as mentioned above, exact geoelectric field values are obtained when $\frac{t_{0}}{T}$ goes to infinity making $F_{C}, F_{S}$, $G_{C}$ and $G_{S}$ equal to $\frac{1}{2}$. Although the general tendency is that the longer the impulse responses the closer the coefficients $F_{C}, F_{S}, G_{C}$ and $G_{S}$ to $\frac{1}{2}$ and thus the better the accuracy of the geoelectric field calculations, the oscillatory character of $F_{C}, F_{S}, G_{C}$ and $G_{S}$ indicates that a small increase of the impulse response length does not necessarily lead to a higher accuracy of the geoelectric field values. Calculation involving a range of frequencies will have some $F_{C}, F_{S}, G_{C}$ and $G_{S}$ values above $\frac{1}{2}$ and some below $\frac{1}{2}$, likely to average out to $\frac{1}{2}$.

We now consider the maxima and minima of $F_{C}, F_{S}, G_{C}$ and $G_{S}$ seen in Fig. 2. It follows from the definitions of the Fresnel Integrals (equations (33) and (34)) that the derivatives $\frac{d F_{C}(\eta)}{d \eta}$ and $\frac{d F_{S}(\eta)}{d \eta}$ are $\cos \left(\frac{\pi \eta^{2}}{2}\right)$ and $\sin \left(\frac{\pi \eta^{2}}{2}\right)$, respectively. The maxima and minima of any function occur at points where the derivative is zero, so the locations of the maxima and minima of the Fresnel Integrals are found by solving the equations

$$
\begin{aligned}
& \cos \left(\frac{\pi \eta^{2}}{2}\right)=0 \\
& \sin \left(\frac{\pi \eta^{2}}{2}\right)=0
\end{aligned}
$$

Equation (43) leads to the solution $\frac{\pi \eta^{2}}{2}=\frac{\pi}{2}+n \pi \quad(n=$ integer $)$, i.e. $\eta=\sqrt{2 n+1}$, and from equation (44) we obtain $\frac{\pi \eta^{2}}{2}=n \pi \quad(n=$ integer $)$, i.e. $\eta=\sqrt{2 n}$. Consequently, $F_{C}(\eta)$ with $\eta=2 \sqrt{\frac{t_{0}}{T}}$ gets its maxima and minima when

$$
\frac{t_{0}}{T}=\frac{n}{2}+\frac{1}{4} \quad(n=\text { integer })
$$

and $F_{S}(\eta)$ with $\eta=2 \sqrt{\frac{t_{0}}{T}}$ gets its maxima and minima when

$$
\frac{t_{0}}{T}=\frac{n}{2} \quad(n=\text { integer })
$$


It can be demonstrated that when $F_{C}(\eta)$ reaches a maximum or minimum, $F_{S}(\eta)$ is close to (but not exactly) $\frac{1}{2}$, and similarly, when $F_{S}(\eta)$ reaches a maximum or minimum, $F_{C}(\eta)$ is close to (but not exactly) $\frac{1}{2}$. This behaviour is related to the facts that cos gets its maxima and minima when $\sin$ is zero, and vice versa, and that the Fresnel Integrals involve integrating cos and sin. The observation that the values are not exactly $\frac{1}{2}$ is due to the fact that the integration variable $s$ is squared in equations (33) and (34).

Using formulas (41) and (42), a simple calculation shows that the derivatives $\frac{d G_{c}(\eta)}{d \eta}$ and $\frac{d G_{s}(\eta)}{d \eta}$ are $\frac{1}{\pi \eta^{2}} \sin \left(\frac{\pi \eta^{2}}{2}\right)$ and $-\frac{1}{\pi \eta^{2}} \cos \left(\frac{\pi \eta^{2}}{2}\right)$, respectively. This means that $G_{C}(\eta)$ gets its maxima and minima when $\sin \left(\frac{\pi \eta^{2}}{2}\right)=0$ and $G_{S}(\eta)$ gets its maxima and minima when $\cos \left(\frac{\pi \eta^{2}}{2}\right)=0$. Referring to the investigation of $F_{C}$ and $F_{S}$, we then see that $G_{C}$ has the maxima and minima at the same points as those of $F_{S}$, i.e. when equation (46) is satisfied, and the maxima and minima of $G_{S}$ occur at the same points as those of $F_{C}$, i.e. when equation (45) is satisfied.

At the maxima and minima of $G_{C}$, the second term on the right-hand side of formula (41) is zero making $G_{C}$ equal to $F_{C}$. As mentioned above, the maxima and minima of $G_{C}$ occur at the same points as those of $F_{S}$, where $F_{C}$ is close to $\frac{1}{2}$. Consequently, $G_{C}$ is close to $\frac{1}{2}$ at its maxima and minima, which means that $G_{C}$ is always close to $\frac{1}{2}$ (except for the very small values of $\frac{t_{0}}{T}$, see Fig. 2). Similarly, at the maxima and minima of $G_{S}$, the second term on the right-hand side of formula (42) is zero making $G_{S}$ equal to $F_{S}$. Since the maxima and minima of $G_{S}$ occur at the same points as those of $F_{C}$, where $F_{S}$ is close to $\frac{1}{2}, G_{S}$ is close to $\frac{1}{2}$ at its maxima and minima, which means that $G_{S}$ is always close to $\frac{1}{2}$ (except for the very small values of $\frac{t_{0}}{T}$, see Fig. 2).

In conclusion, what is most important to note from the above investigation of $F_{C}, F_{S}, G_{C}$ and $G_{S}$ is that $G_{C}$ and $G_{S}$ approach $\frac{1}{2}$ much faster than $F_{C}$ and $F_{S}$ when $\frac{t_{0}}{T}$ increases. This means that, using $B(t)$ and the high-pass impulse response, a much shorter impulse response can be applied, compared to using $\frac{d B(t)}{d t}$ and the low-pass impulse response, to obtain the same accuracy of the geoelectric field. This will be investigated more in Section 6 by using a test geomagnetic variation waveform.

\section{Calculations Using a Test Geomagnetic Variation Waveform}

We now study the effect of the truncation of the impulse responses on the calculated geoelectric field values by using an artificial, but realistic, test geomagnetic variation waveform consisting of six sinusoidal waves and expressed by

$$
B(t)=\sum_{k=1}^{6} B_{k} \sin \left(2 \pi f_{k} t+\phi_{k}\right)
$$

where the time $t$, in principle, extends from $-\infty$ to $+\infty$. The amplitudes $B_{k}$, the frequencies $f_{k}$ and the phase angles $\phi_{k}$ are given in Table 1 , which also shows the periods $T_{k}\left(=\frac{1}{f_{k}}\right)$. The amplitudes $B_{k}$ are approximately inversely proportional to the frequencies $f_{k}$, which roughly corresponds to the spectrum of 
real geomagnetic field variations. The phase angles $\phi_{k}$ are chosen arbitrarily. A three-day sample (starting at $t=0$ ) of the geomagnetic field variation given by formula (47) is shown in Fig. 3.

Table 1. Parameter values of an artificial test geomagnetic variation waveform defined by equation (47). These values are used in all analyses in Section 6.

\begin{tabular}{ccccc}
\hline $\boldsymbol{k}$ & $\boldsymbol{B}_{\boldsymbol{k}}[\mathbf{n T}]$ & $\square \phi_{k}[\mathrm{deg}]$ & $\boldsymbol{f}_{\boldsymbol{k}}[\mathbf{H z}]$ & $\boldsymbol{T}_{\boldsymbol{k}}=\mathbf{1} / \boldsymbol{f}_{\boldsymbol{k}}[\mathbf{m i n}]$ \\
\hline 1 & 200 & 10 & 0.00009259 & 180 \\
2 & 90 & 20 & 0.00020833 & 80 \\
3 & 30 & 30 & 0.00047619 & 35 \\
4 & 17 & 40 & 0.00111111 & 15 \\
5 & 8 & 50 & 0.00238095 & 7 \\
6 & 3.5 & 60 & 0.00555555 & 3 \\
\hline
\end{tabular}

Test Geomagnetic Variation, Six Sine Functions

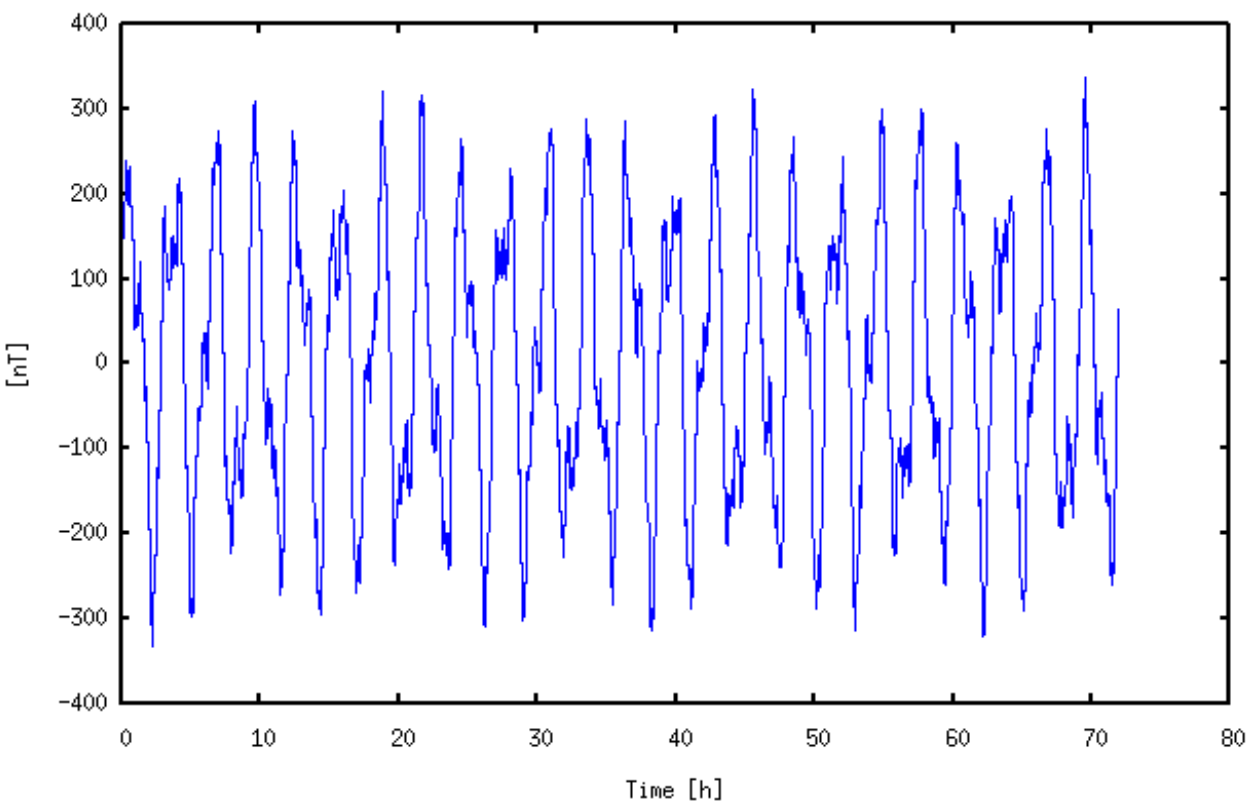

Figure 3. Test geomagnetic field variation on three days (starting at the time $t=0$ ) obtained from equation (47) with the parameter values given in Table 1 .

For simplicity, we again use a model of the Earth with uniform conductivity $\sigma$. We believe that this assumption does not prevent us from drawing conclusions also valid for more complicated Earth conductivity structures but, as seen in Section 5, this assumption enables the derivation of an exact analytic expression for the geoelectric field (see equation (30)). Thus, noting that the amplitudes $B_{k}$ correspond to $B_{0}$ used in Section 5 and that the angular frequency $\omega_{k}$ is related to the frequency $f_{k}$ by $\omega_{k}=2 \pi f_{k}$, the exact expression of the geoelectric field induced by the geomagnetic field variation given by equation (47) is

$$
E_{\text {exact }}(t)=\sum_{k=1}^{6} B_{k} \sqrt{\frac{2 \pi f_{k}}{\mu_{0} \sigma}} \sin \left(2 \pi f_{k} t+\phi_{k}+\frac{\pi}{4}\right)
$$

Using a value of $0.001 \mathrm{~S} / \mathrm{m}$ for the Earth's conductivity, formula (48) gives the geoelectric field shown in Fig. 4 for three days starting at $t=0$. 
Exact Analytic Geoelectric Field, Uniform Earth 1000 ohm m

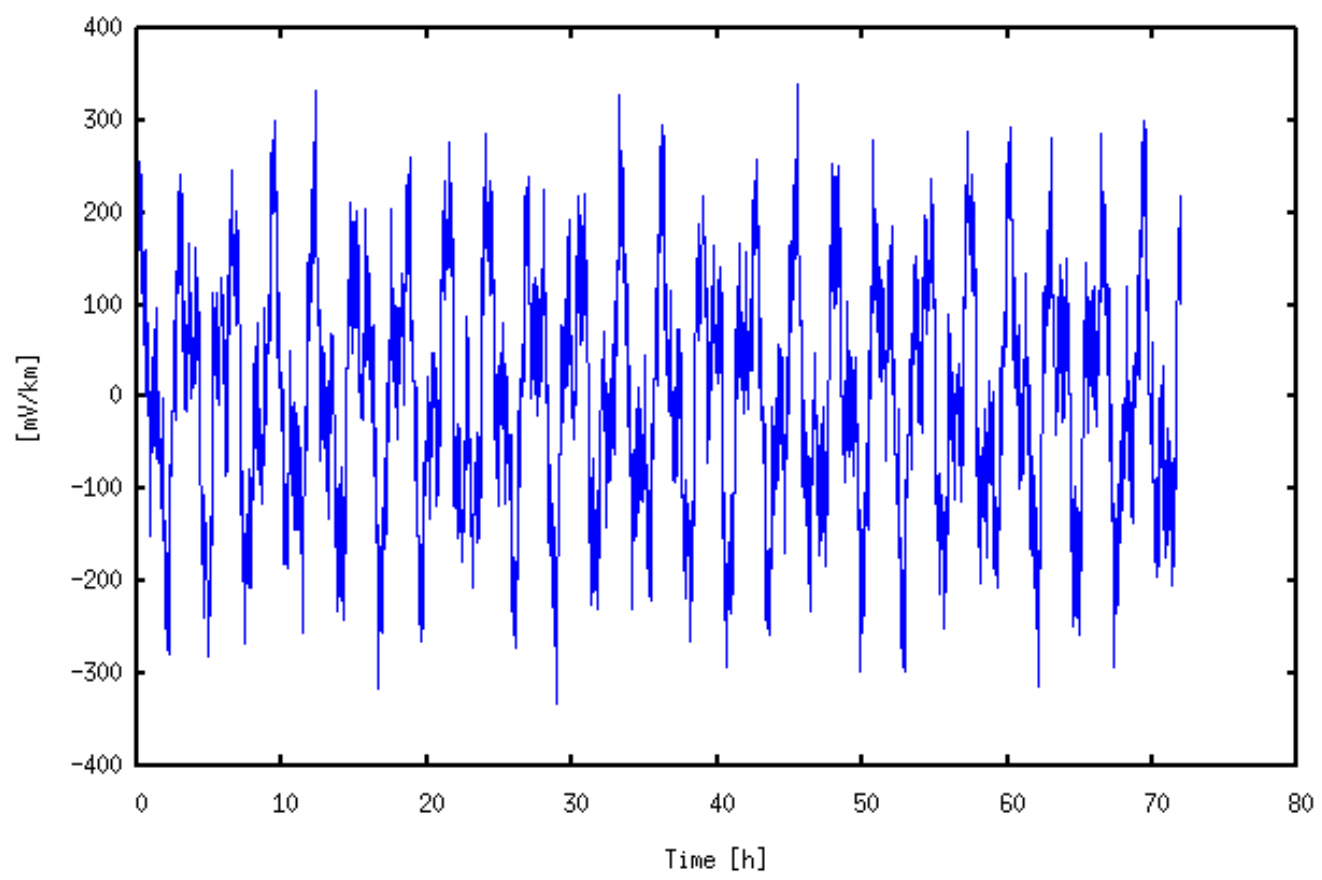

Figure 4. Exact geoelectric field on three days (starting at the time $t=0$ ) calculated using equation (48) with the parameter values given in Table 1 and assuming that the Earth's conductivity is $\sigma=0.001 \mathrm{~S} / \mathrm{m}$.

The expressions of the approximate geoelectric fields $E_{\text {trunc_B }}(t)$ and $E_{\text {trunc_g }}(t)$ obtained by applying truncated high-pass and low-pass impulse responses having the length $t_{0}$ can be derived using equations (40) and (36), respectively, which gives

$$
\begin{aligned}
& E_{\text {trunc_B }}(t)=\sum_{k=1}^{6} 2 B_{k} \sqrt{\frac{\pi f_{k}}{\mu_{0} \sigma}}\left[G_{C}^{k} \cos \left(2 \pi f_{k} t+\phi_{k}\right)+G_{S}^{k} \sin \left(2 \pi f_{k} t+\phi_{k}\right)\right] \\
& E_{\text {trunc_g }}(t)=\sum_{k=1}^{6} 2 B_{k} \sqrt{\frac{\pi f_{k}}{\mu_{0} \sigma}}\left[F_{C}^{k} \cos \left(2 \pi f_{k} t+\phi_{k}\right)+F_{S}^{k} \sin \left(2 \pi f_{k} t+\phi_{k}\right)\right]
\end{aligned}
$$

where $F_{C}^{k}$ and $F_{S}^{k}$ are the Fresnel Cosine and Sine Integrals defined by equations (33) and (34) with $\xi=2 \sqrt{f_{k} t_{0}}$, and the coefficients $G_{C}^{k}$ and $G_{S}^{k}$ are obtained from equations (41) and (42). Thus

$$
\begin{aligned}
F_{C}^{k} & =\int_{0}^{2 \sqrt{f_{k} t_{0}}} \sin \left(\frac{\pi s^{2}}{2}\right) d s \\
F_{S}^{k} & =\int_{0}^{2 \sqrt{f_{k} t_{0}}} \sin \left(\frac{\pi s^{2}}{2}\right) d s \\
G_{C}^{k} & =F_{C}^{k}-\frac{\sin \left(2 \pi f_{k} \mathrm{t}_{0}\right)}{2 \pi \sqrt{f_{k} \mathrm{t}_{0}}} \\
G_{S}^{k} & =F_{S}^{k}-\frac{\cos \left(2 \pi f_{k} \mathrm{t}_{0}\right)}{2 \pi \sqrt{f_{k} \mathrm{t}_{0}}}
\end{aligned}
$$


Table 2. Values of the coefficients $F_{C}^{k}, F_{S}^{k}, G_{C}^{k}$ and $G_{S}^{k}$ defined by equations (51), (52), (53) and (54) and used in formulas (49) and (50) for four impulse response lengths $\left(t_{0}=1,4,12,24\right.$ hours) and for the six periods $T_{k}$ given in Table 1.

\begin{tabular}{|c|c|c|c|c|c|c|}
\hline Length $t_{0}[\mathrm{~h}]$ & $T=180 \mathrm{~min}$ & $T=80 \mathrm{~min}$ & $T=35 \mathrm{~min}$ & $T=15 \mathrm{~min}$ & $T=7 \mathrm{~min}$ & $T=3 \mathrm{~min}$ \\
\hline 1 & 0.741 & 0.321 & 0.383 & 0.498 & 0.477 & 0.500 \\
\hline 4 & 0.622 & 0.498 & 0.452 & 0.500 & 0.527 & 0.500 \\
\hline 12 & 0.498 & 0.500 & 0.485 & 0.500 & 0.488 & 0.500 \\
\hline 24 & 0.499 & 0.500 & 0.519 & 0.500 & 0.489 & 0.500 \\
\hline \multicolumn{7}{|l|}{$F_{S}^{k}$} \\
\hline Length $t_{0}[\mathrm{~h}]$ & $T=180 \mathrm{~min}$ & $T=80 \mathrm{~min}$ & $T=35 \mathrm{~min}$ & $T=15 \mathrm{~min}$ & $T=7 \mathrm{~min}$ & $T=3 \mathrm{~min}$ \\
\hline 1 & 0.586 & 0.517 & 0.532 & 0.421 & 0.549 & 0.464 \\
\hline 4 & 0.561 & 0.408 & 0.463 & 0.460 & 0.506 & 0.482 \\
\hline 12 & 0.421 & 0.447 & 0.532 & 0.477 & 0.490 & 0.490 \\
\hline 24 & 0.444 & 0.462 & 0.484 & 0.484 & 0.502 & 0.493 \\
\hline \multicolumn{7}{|l|}{$G_{C}^{k}$} \\
\hline Length $t_{0}[\mathrm{~h}]$ & $T=180 \mathrm{~min}$ & $T=80 \mathrm{~min}$ & $T=35 \mathrm{~min}$ & $T=15 \mathrm{~min}$ & $T=7 \min$ & $T=3 \min$ \\
\hline 1 & 0.503 & 0.505 & 0.502 & 0.498 & 0.500 & 0.500 \\
\hline 4 & 0.503 & 0.498 & 0.500 & 0.500 & 0.500 & 0.500 \\
\hline 12 & 0.498 & 0.500 & 0.500 & 0.500 & 0.500 & 0.500 \\
\hline 24 & 0.500 & 0.500 & 0.500 & 0.500 & 0.500 & 0.500 \\
\hline \multicolumn{7}{|l|}{$G_{S}^{k}$} \\
\hline Length $t_{0}[\mathrm{~h}]$ & $T=180 \mathrm{~min}$ & $T=80 \mathrm{~min}$ & $T=35 \mathrm{~min}$ & $T=15 \mathrm{~min}$ & $T=7$ min & $T=3 \mathrm{~min}$ \\
\hline 1 & 0.448 & 0.517 & 0.505 & 0.500 & 0.500 & 0.500 \\
\hline 4 & 0.493 & 0.500 & 0.501 & 0.500 & 0.500 & 0.500 \\
\hline 12 & 0.500 & 0.500 & 0.500 & 0.500 & 0.500 & 0.500 \\
\hline 24 & 0.500 & 0.500 & 0.500 & 0.500 & 0.500 & 0.500 \\
\hline
\end{tabular}

Using equations (49) and (50), we compute $E_{\text {trunc_B }}(t)$ and $E_{\text {trunc } g}(t)$ during the three-day sequence included in Figures 3 and 4. Four different impulse response lengths are considered, which are $t_{0}=1,4$, 12, 24 hours. The values of the coefficients $F_{C}^{k}, F_{S}^{k}, G_{C}^{k}$ and $G_{S}^{k}$ used in formulas (49) and (50) are given in Table 2. Comparisons of $E_{\text {trunc } B}(t)$ and $E_{\text {trunc }}(t)$ with $E_{\text {eract }}(t)$ given by equation (48) are made for the geoelectric field values on the middle day of the three-day sequence included in Figures 3 and 4 , i.e. from $1440 \mathrm{~min}$ to $2879 \mathrm{~min}$, thus including 1440 data points with a one-minute sampling interval.

Figures 5-8 show the approximate geoelectric field based on a truncated impulse response on the middle day (1440 red data points) as a function of the exact geoelectric field given by formula (48). The Earth's conductivity is set to $\sigma=0.001 \mathrm{~S} / \mathrm{m}$. The unit of the geoelectric fields is $[\mathrm{mV} / \mathrm{km}]$ in the figures. The black lines show the least-square fits as follows

$$
E_{\text {trunc }}=a E_{\text {exact }}+b
$$

Figures 5 and 7 refer to "High-Pass" calculations using equation (49) with the impulse response lengths of $1 \mathrm{~h}$ (Fig. 5) and $24 \mathrm{~h}$ (Fig. 7). Figures 6 and 8 depict the results from "Low-Pass" calculations using equation (50) with the impulse response lengths of $1 \mathrm{~h}$ (Fig. 6) and $24 \mathrm{~h}$ (Fig. 8). 


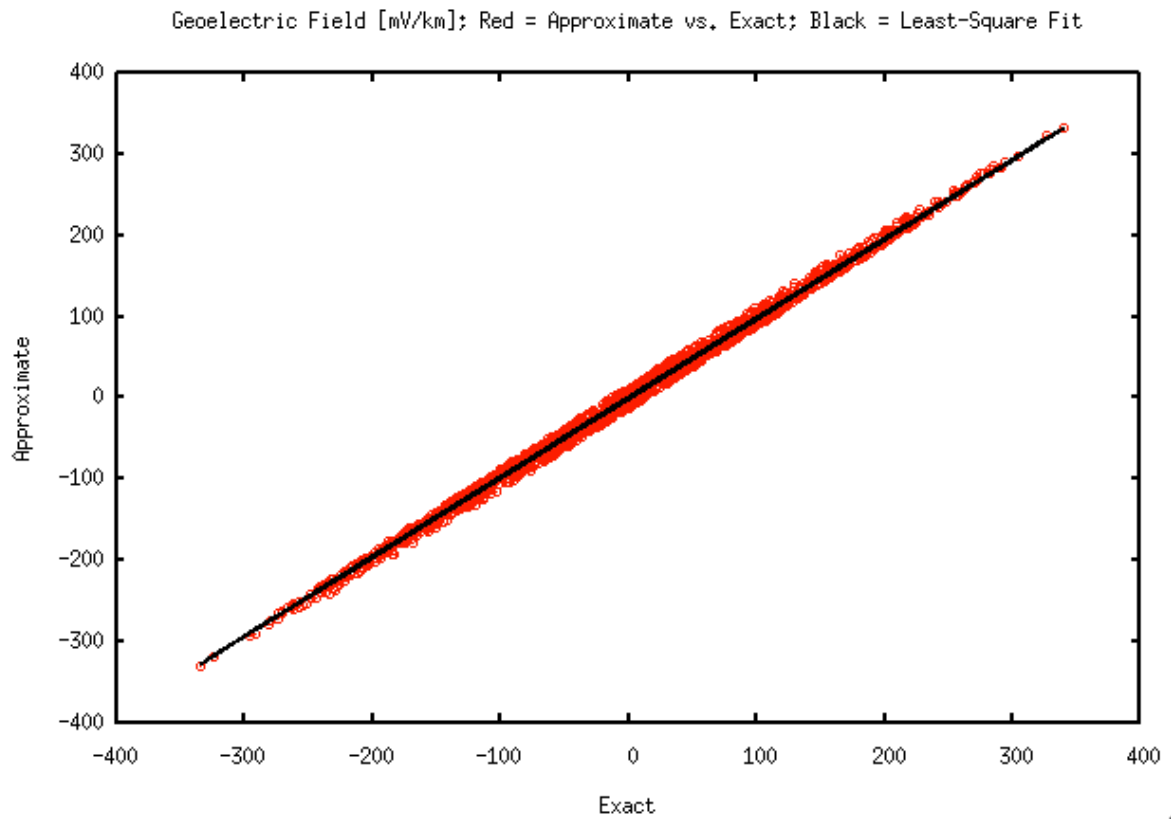

Figure 5. Approximate geoelectric field (vertical axis) obtained using the truncated impulse response as a function of the exact geoelectric field (horizontal axis). The unit on both axes is $[\mathrm{mV} / \mathrm{km}]$. The approximate calculation is performed using the "High-Pass" formula (49) with the impulse response length $t_{0}=1 \mathrm{~h}$. The exact geoelectric field is obtained from equation (48). One-minute values on the middle day of the three-day sequence included in Figures 3 and 4 are considered, so the number of the red data points is 1440 . The black line shows the least-square fit. The values of the parameters included in equations (48) and (49) are given in Tables 1 and 2, and the Earth's conductivity is $\sigma=0.001 \mathrm{~S} / \mathrm{m}$.

Geoelectric Field $[\mathrm{mW} / \mathrm{km}] ;$ Red = Approximate vs. Exact; Black = Least-Square Fit

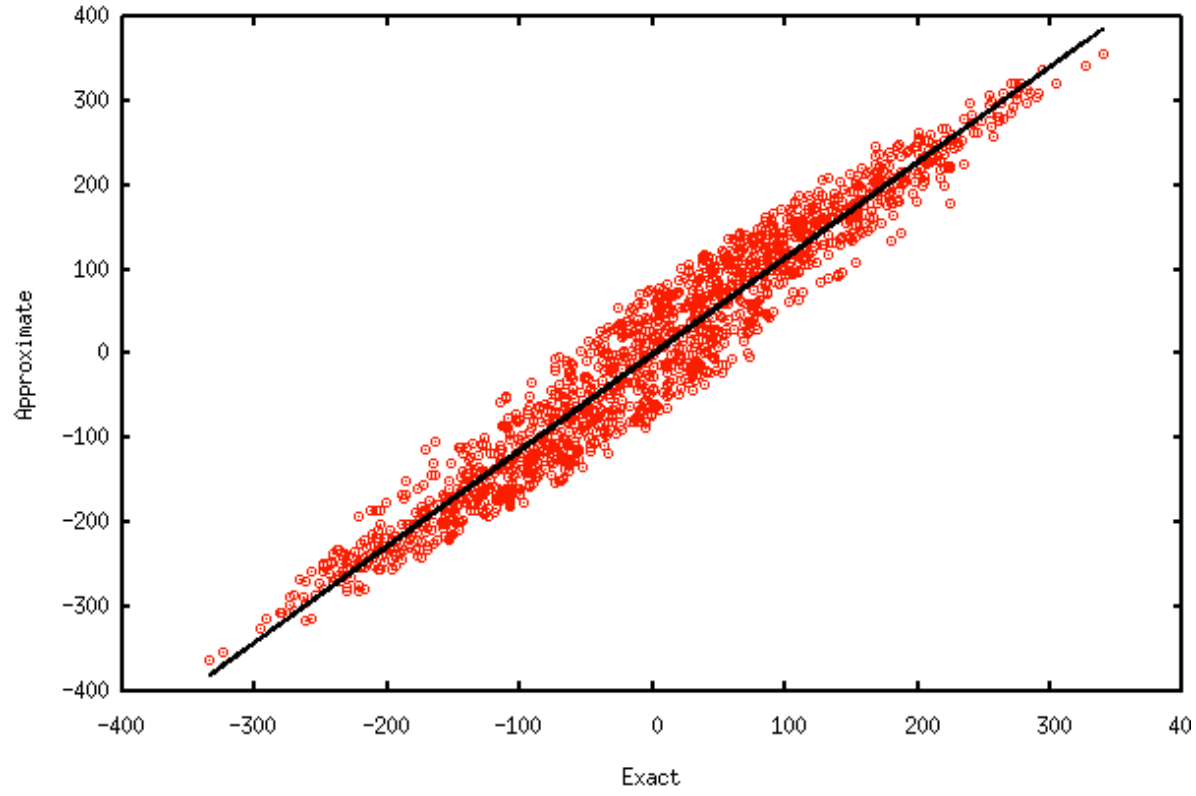

Figure 6. Similar to Fig. 5 but the approximate calculation is based on the "Low-Pass" formula (50) with the impulse response length $t_{0}=1 \mathrm{~h}$. The values of the parameters included in equation (50) are given in Tables 1 and 2 , and the Earth's conductivity is $\sigma=0.001 \mathrm{~S} / \mathrm{m}$. 
Geoelectric Field $[\mathrm{m} / \mathrm{km}]$; Red = Approximate vs, Exact; Black = Least-Square Fit

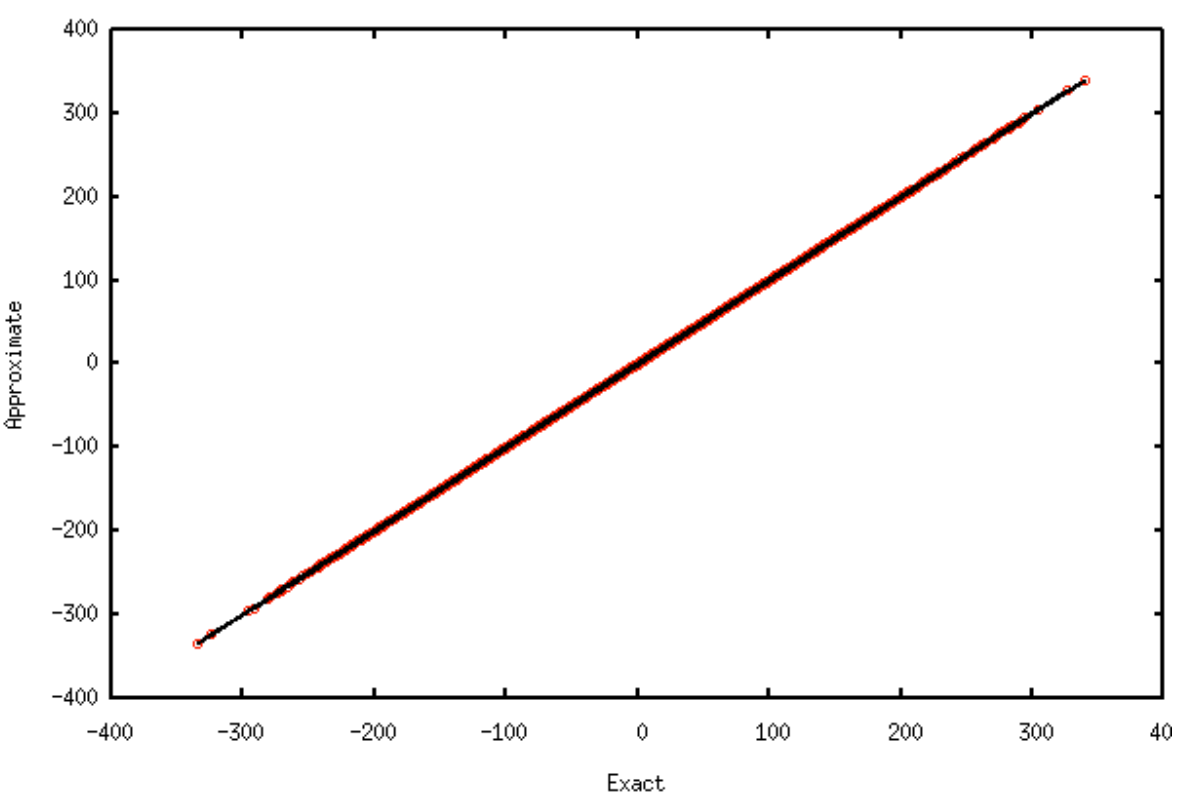

Figure 7. Similar to Fig. 5 but the impulse response length is $t_{0}=24 \mathrm{~h}$.

Geoelectric Field $[\mathrm{m} W / \mathrm{km}] ;$ Red = Approximate vs, Exact; Black = Least-Square Fit

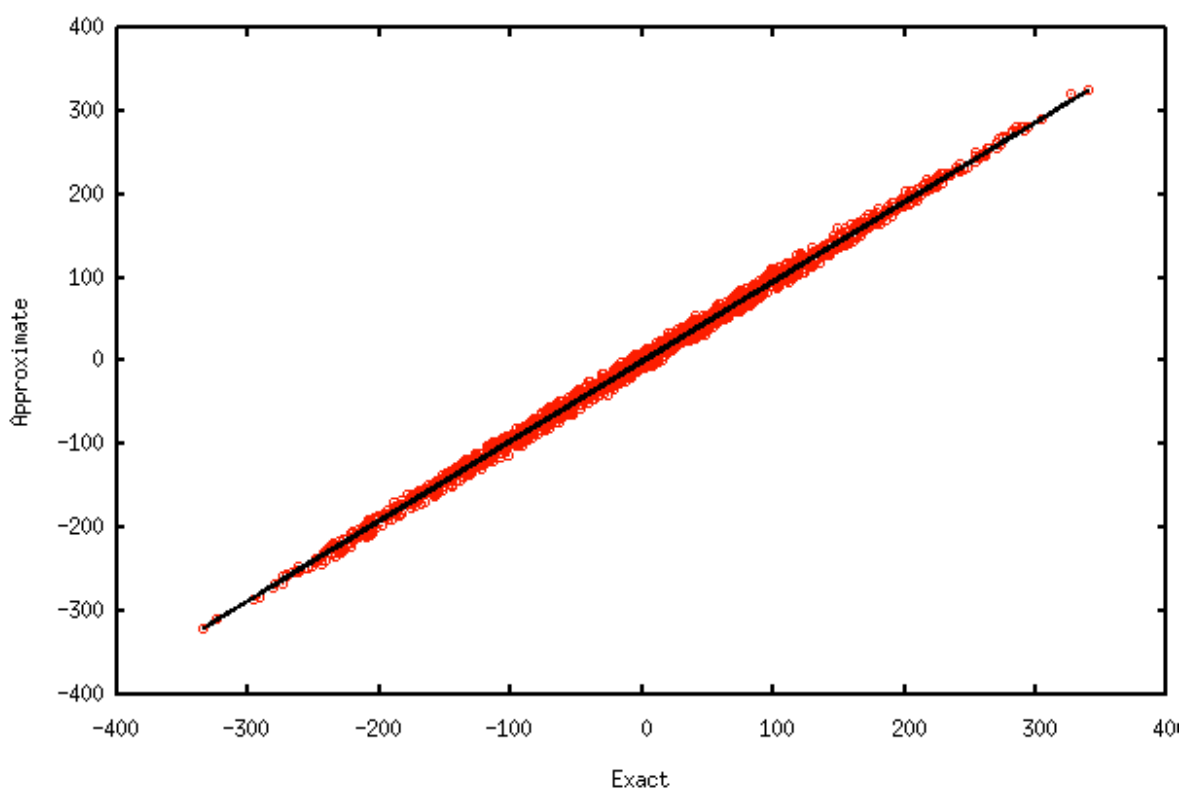

Figure 8. Similar to Fig. 6 but the impulse response length is $t_{0}=24 \mathrm{~h}$.

Table 3 contains a summary of the comparisons between $E_{\text {exact }}(t)$ and $E_{\text {trunc_B }}(t)$ ("High-Pass") or $E_{\text {trunc_g }}(t)$ ("Low-Pass") including all four impulse response lengths considered. The parameters $a$ and $b$ give the least-square fits of the 1440 data points according to equation (55). In Table 3, all correlation coefficients between the exact geoelectric field and the approximate geoelectric fields obtained using truncated impulse responses are very high, and all values of $a$ and $b$ are close to one and zero, respectively. Consequently, the agreement between the exact and approximate geoelectric field values is 
very good in all cases considered. However, looking at the numbers included in Table 3 more closely, we can see that the "High-Pass" values refer to an even better agreement than the "Low-Pass" values and that the "High-Pass" values vary less when the impulse response length changes. This indicates that, in numerical calculations based on the high-pass impulse response $R_{\text {trunc }}(t)$ and $B(t)$, the truncation of the convolution integral can be made much earlier than when using the low-pass impulse response $S_{\text {trunc }}(t)$ and $g(\mathrm{t})=\frac{d B(\mathrm{t})}{d t}$, and even the length of one hour would be sufficient in the case of $R_{\text {trunc }}(t)$ and $B(t)$. As discussed in Section 4, the reason for this is that $R(t)$ given by equation (24) goes to zero with increasing $t$ much faster than $S(t)$ given by equation (23) (see Fig. 1). These observations about shorter impulse response lengths in "High-Pass" calculations than in "Low-Pass" calculations are also in agreement with the conclusions drawn in Section 5.

Figures 5 and 8 look very similar, and the corresponding (i.e. "High-Pass" - 1 h and "Low-Pass - 24 h) values of the correlation coefficient are near each other in Table 3. Moreover, the values of the parameter $a$ do not differ much in these two cases in Table 3. (The values of the parameter $b$ differ relatively more but they are insignificant anyway.) Consequently, we may conclude that in "High-Pass" calculations the same accuracy of the geoelectric field is already obtained with an impulse response length of one hour that requires the length of twenty-four hours in "Low-Pass" calculations. The large scatter in Fig. 6 supports the conclusion that the length of one hour is definitely too short in "LowPass" calculations. As expected, "High-Pass" calculations with an impulse response having the length of $24 \mathrm{~h}$ lead to very precise geoelectric results as seen from Fig. 7, which shows no scatter.

Figures 9-11 present the approximate geoelectric field obtained using the truncated impulse response (blue) and the exact geoelectric field given by formula (48) (red) during four hours in the beginning of the middle day of the three-day sequence included in Figures 3 and 4. The Earth's conductivity is again set to $\sigma=0.001 \mathrm{~S} / \mathrm{m}$. Figure 9 refers to a "High-Pass" calculation using equation (49) with the impulse response length of $1 \mathrm{~h}$. Figures 10 and 11 show the results from "Low-Pass" calculations using equation (50) with the impulse response lengths of $1 \mathrm{~h}$ (Fig. 10) and $24 \mathrm{~h}$ (Fig. 11).

Table 3. Correlation coefficients and least-square fit parameters $a$ and $b$ (equation (55)) between the exact geoelectric field (equation (48)) and the geoelectric fields obtained using truncated impulse responses (equation (49) - "High-Pass" and equation (50) - "Low-Pass"). Four impulse response lengths are considered: $t_{0}=1,4,12,24$ hours. The analysis concerns one-minute values on the middle day of the three-day sequence included in Figures 3 and 4, i.e. 1440 data points. The values of the parameters included in equations (48), (49) and (50) are given in Tables 1 and 2, and the Earth's conductivity is $\sigma=0.001 \mathrm{~S} / \mathrm{m}$.

High-Pass

\begin{tabular}{cccc}
\hline Length $\boldsymbol{t}_{\boldsymbol{0}}[\mathrm{h}]$ & Corr.Coeff & $\boldsymbol{a}$ & $\boldsymbol{b}[\mathrm{mV} / \mathrm{km}]$ \\
\hline 1 & 0.99855 & 0.978 & 0.0037 \\
4 & 0.99997 & 0.997 & 0.0005 \\
12 & 0.9999990 & 0.999 & 0.0001 \\
24 & 0.99999989 & 1.000 & 0.0000 \\
\hline & & & \\
Low-Pass & & & \\
\hline Length $\boldsymbol{t}_{\boldsymbol{o}}[\mathrm{h}]$ & Corr.Coeff & $\boldsymbol{a}$ & $\boldsymbol{b}[\mathrm{mV} / \mathrm{km}]$ \\
\hline 1 & 0.97153 & 1.138 & -0.0031 \\
4 & 0.99092 & 1.075 & -0.0137 \\
12 & 0.99704 & 0.938 & 0.0141 \\
24 & 0.99857 & 0.956 & 0.0007 \\
\hline
\end{tabular}


Geoelectric Fieldः Red=E_exact, Blue=E_trunc_B; to $=1 \mathrm{~h}$

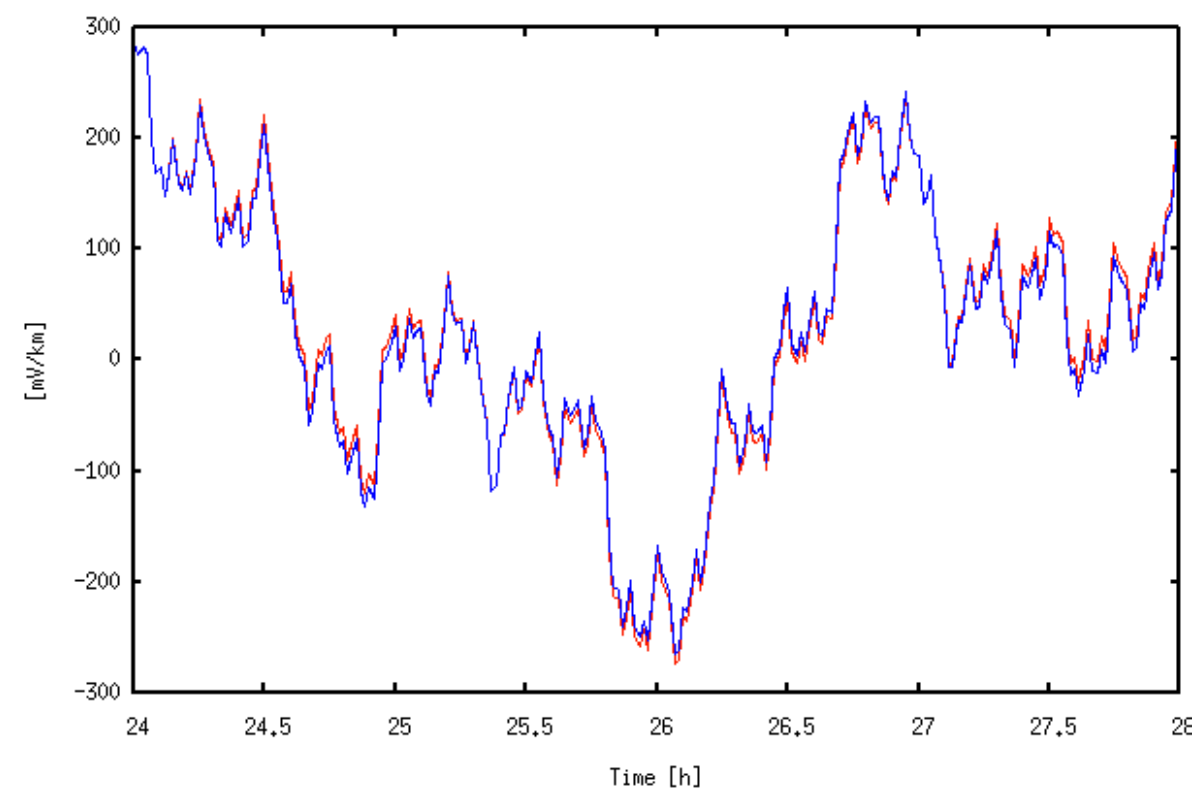

Figure 9. Approximate geoelectric field obtained using the truncated impulse response (blue) and exact geoelectric field (red) during four hours in the beginning of the middle day included in Figures 3 and 4 . The approximate calculation is performed using the "High-Pass" formula (49) with the impulse response length $t_{0}=1 \mathrm{~h}$. The exact geoelectric field is obtained from equation (48). The values of the parameters included in equations (48) and (49) are given in Tables 1 and 2, and the Earth's conductivity is $\sigma=0.001 \mathrm{~S} / \mathrm{m}$.

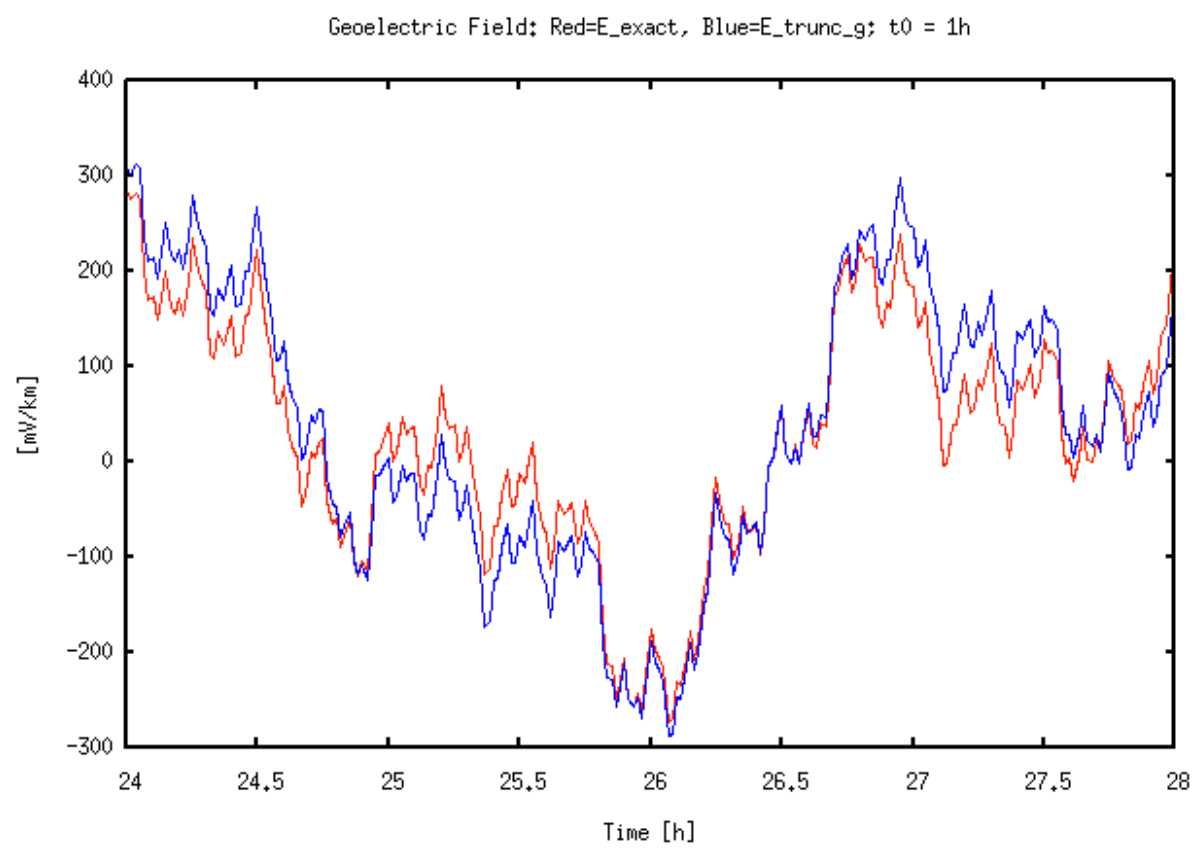

Figure 10. Similar to Fig. 9 but the approximate calculation is based on the "Low-Pass" formula (50) with the impulse response length $t_{0}=1 \mathrm{~h}$. The values of the parameters included in equation (50) are given in Tables 1 and 2 , and the Earth's conductivity is $\sigma=0.001 \mathrm{~S} / \mathrm{m}$. 


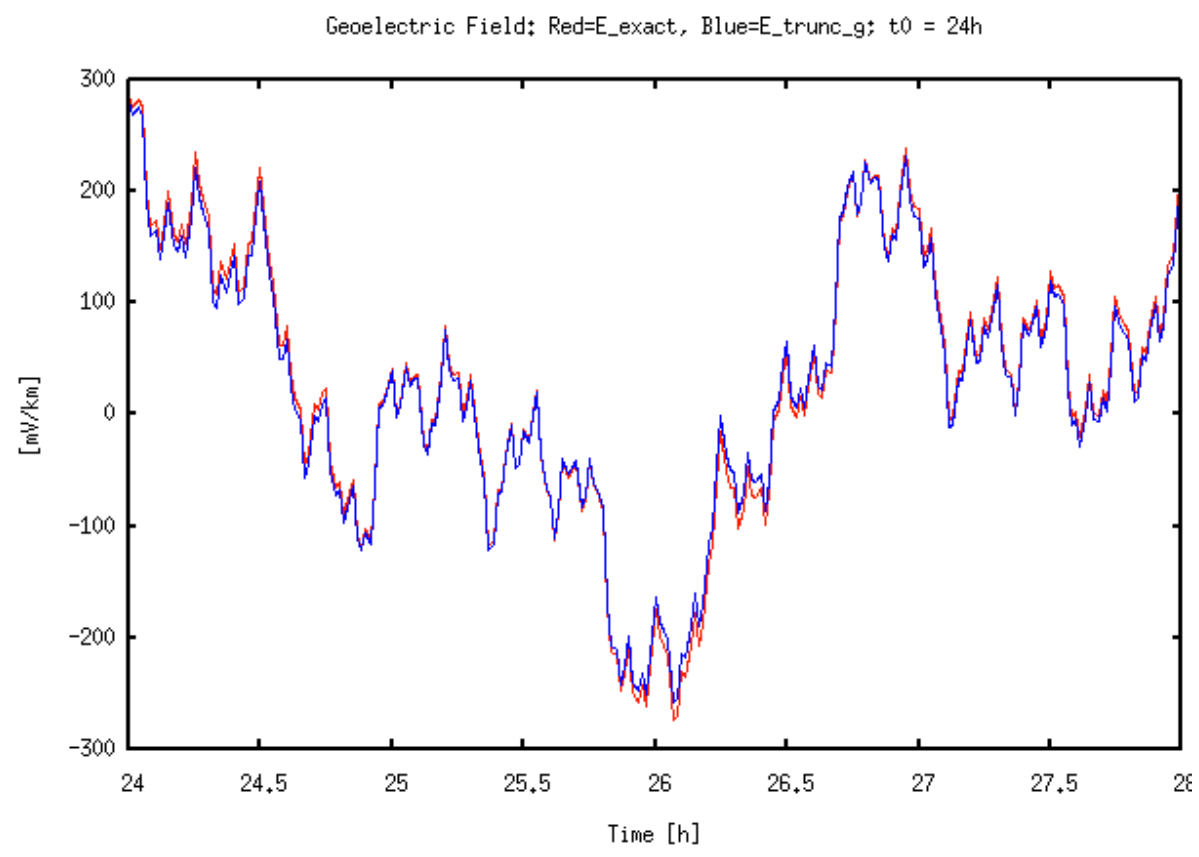

Figure 11. Similar to Fig. 10 but the impulse response length is $t_{0}=24 \mathrm{~h}$.

Comparison of Figures 9 and 11 supports the conclusion drawn from Figures 5 and 8 above that "High-Pass" calculations lead to the same accuracy with an impulse response length of $1 \mathrm{~h}$ as "LowPass" calculations with an impulse response length of $24 \mathrm{~h}$. The large differences between the blue and red curves in Fig. 10 again emphasise that $1 \mathrm{~h}$ is clearly too short an impulse response length in "LowPass" calculations. Looking carefully, we may see some, though insignificant, differences between the blue and red curves in Fig. 9, but if the impulse response length is extended to $4 \mathrm{~h}$ in "High-Pass" calculations it is impossible to see any differences between the two curves (not shown in this paper).

\section{Conclusion}

In the time domain, the geoelectric field can be expressed as a convolution between the geomagnetic field variation or its time derivative and an impulse response function. The geomagnetic field variation is convolved with a high-pass impulse response and the time derivative of the geomagnetic variation with a low-pass impulse response. Mathematically the convolution integrals extend to infinity but in practical calculations only finite intervals can be used, i.e. the impulse responses are truncated.

The effect of the truncation on the geoelectric field is investigated in this paper. For a sinusoidal geomagnetic field variation and a uniform-conductivity Earth model, it is possible to derive an analytic expression for the exact geoelectric field and analytic expressions for the approximate geoelectric field as functions of the truncated impulse response length using the Fresnel Cosine and Sine Integrals. These expressions enable studying the effect of the impulse response length on the accuracy of the geoelectric field values in detail.

The calculations discussed in this paper utilise an artificial test geomagnetic variation waveform consisting of six sinusoidal waves and response functions calculated for an earth model with uniform conductivity. It is seen that the high-pass impulse response used for convolution with geomagnetic data can be truncated quite early. Even the length of one hour is enough to provide accurate geoelectric field values. But, in the convolution with the time derivative of the geomagnetic field, the low-pass impulse response must be extended until several hours, even to twenty-four hours. This can be understood mathematically by the slower decrease of the low-pass impulse response with increasing time lag between geoelectric and geomagnetic data.

Although the studies discussed in this paper use an Earth model with uniform conductivity the conclusions about the impulse response lengths are certainly qualitatively true for non-uniform Earth 
models as well. As analytic expressions of the impulse responses and of the geoelectric field are not available in non-uniform cases, quantitative conclusions about the accuracy of the geoelectric field values would require precise and detailed numerical calculations using the particular Earth models.

\section{References}

1. P. R. Gattens, "Investigation of transformer overheating due to solar magnetic disturbances," IEEE Power Engineering Review, October 1989, pp. 19-20, 1989.

2. C. T. Gaunt and G. Coetzee, "Transformer failures in regions incorrectly considered to have low GIC-risk," PowerTech'07 Conference, Lausanne, Switzerland, 1-5 July 2007, Paper n:o 445 at the 6 pp., 2007.

3. J. G. Kappenman, "Geomagnetic disturbances and impacts upon power system operation," In: The Electric Power Engineering Handbook, L.L., Grigsby (Ed.), 2nd Edn., Chapter 16, pp. 16-1 - 16-22, CRC Press/IEEE Press, 2007.

4. T. S. Molinski, "Why utilities respect geomagnetically induced currents," Journal of Atmospheric and SolarTerrestrial Physics, vol. 64, no. 16, pp. 1765-1778, 2002.

5. B. Bozoki, S. R. Chano, W. E. Feero, G. Fenner, C. F. Henville, J. W. Ingleson, S. Mazumdar, P. G. McLaren, K. K. Mustaphi, F. M. Phillips, and G. D. Rockefeller, "The effects of GIC on protective relaying," IEEE Transactions on Power Delivery, vol. 11, no. 2, pp. 725-739, doi: 10.1109/61.489329, 1996.

6. T. Maguire and D. Woodford, "Geomagnetic induced current effect on SVC operation," CEA Report, 316 T 745, 1990.

7. NERC GMDTF 2012 Special Reliability Assessment Interim Report, "Effects of Geomagnetic Disturbances on the Bulk Power System," North American Electricity Reliability Corporation (NERC), 150 pp., 2012.

8. R. Pirjola, "Review on the calculation of surface electric and magnetic fields and of geomagnetically induced currents in ground-based technological systems," Surveys in Geophysics, vol. 23, no. 1, pp. 71-90, 2002.

9. D. Boteler, "On Choosing Fourier Transforms for Practical Geoscience Applications," International Journal of Geosciences, vol. 3, pp. 952-959, doi: 10.4236/ijg.2012.325096, 2012.

10. D. H. Boteler, "Geomagnetically induced currents: Present knowledge and future research," IEEE Transactions on Power Delivery, vol. 9, no. 1, pp. 50-58, 1994.

11. L. Trichtchenko and D. H. Boteler, "Modelling of geomagnetic induction in pipelines," Annales Geophysicae, vol. 20, no. 7, pp. 1063-1072, doi: 10.5194/angeo-20-1063-2002, 2002.

12. D. H. Boteler and R. J. Pirjola, "The complex-image method for calculating the magnetic and electric fields produced at the surface of the Earth by the auroral electrojet," Geophysical Journal International, vol. 132, no. 1, pp. 31-40, 1998.

13. R. Pirjola, "Electromagnetic induction in the earth by a plane wave or by fields of line currents harmonic in time and space," Geophysica, vol. 18, nos. 1-2, pp. 1-161, 1982.

14. R. Pirjola, "Estimation of the electric field on the Earth's surface during a geomagnetic variation," Geophysica, vol. 20, no. 2, pp. 89-103, 1984. 